\title{
Archives
}

\section{Index du volume 47}

VIVIANNE MARÉCHAL

Volume 47, numéro 2, 2018

URI : https://id.erudit.org/iderudit/1045173ar

DOI : https://doi.org/10.7202/1045173ar

Aller au sommaire du numéro

Éditeur(s)

Association des archivistes du Québec (AAQ)

ISSN

0044-9423 (imprimé)

2369-9256 (numérique)

Découvrir la revue

Citer ce document

MARÉCHAL, V. (2018). Index du volume 47. Archives, 47(2), 107-112.

https://doi.org/10.7202/1045173ar

Ce document est protégé par la loi sur le droit d'auteur. L'utilisation des services d'Érudit (y compris la reproduction) est assujettie à sa politique d'utilisation que vous pouvez consulter en ligne.

https://apropos.erudit.org/fr/usagers/politique-dutilisation/
Cet article est diffusé et préservé par Érudit.

Érudit est un consortium interuniversitaire sans but lucratif composé de l’Université de Montréal, l'Université Laval et l'Université du Québec à Montréal. Il a pour mission la promotion et la valorisation de la recherche. https://www.erudit.org/fr/ 


\title{
INDEX
}

\section{Index du volume 47}

\section{VIVIANNE MARÉCHAL}

\author{
Archiviste, Université du Québec à Montréal \\ Directrice de la revue Archives
}

L'index du volume 47 de la revue Archives, publié en 2017 et 2018, se divise en trois parties: un index de chaque auteur de tout article ou compte rendu, un index alphabétique des sujets ainsi qu'un index des ouvrages ayant fait l'objet d'un compte rendu critique. Un ouvrage écrit par plusieurs auteurs se retrouve indexé à chaque auteur. De plus, chaque article est indexé de manière à ce que sa notice bibliographique apparaisse une seule fois dans l'index des sujets. L'index des sujets s'harmonise avec les sujets de la bibliographie annuelle telle que développée en 2017 par David Rajotte, responsable de la bibliographie annuelle de la revue Archives. Finalement, les ouvrages recensés se retrouvent dans l'index des auteurs, sous le nom de l'auteur du compte rendu, ainsi que dans l'index des ouvrages recensés sous le nom de l'auteur de l'ouvrage. 


\section{INDEX DES AUTEURS}

ALAOUI, S. [Compte rendu de I'ouvrage La gestion intégrée des documents d'activité (GID) technologiques et en format papier de M. Roberge]. 47(2), 91-94.

ALAOUI, S. La normalisation et la gestion intégrée des documents (GID): quelle relation? Réflexion sur les normes ISO 30300, ISO 30301, ISO 14641 et leur apport à l'implantation des systèmes de GID. 47(1), 7-30.

ASSOCIATION DES ARCHIVISTES DU QUÉBEC (AAQ). Renouvellement de la politique culturelle du Québec. 47(2), 49-58.

BAILLARGEON, D. Les centres et les services d'archives du Québec se font tirer le portrait... statistique. Quelques considérations méthodologiques et résultats d'une préenquête. 47(1), 125-152.

BRÉARD, J. [Compte rendu de I'ouvrage Archives hospitalières. Regards croisés de S. Monnier et K. Fiorentino]. 47(2), 95-98.

BROCHU, S. [Compte rendu de l'ouvrage Amateur Filmmaking: The Home Movie, the Archive, the Web de L. Rascaroli, G. Young et B. Monahan]. 47(1), 185-191.

CARTIER, F. Le traitement et la diffusion du fonds Armand-Frappier (ou comment être opportuniste en huit leçons). 47(2), 7-20.

CHALIFOUR, J. La subjectivité dans la numérisation: les perspectives des professionnels. 47(1), 31-58.

COMITÉ DES ARCHIVES DU CONSEIL DU PATRIMOINE RELIGIEUX DU QUÉBEC (CPRQ). Forces vives oubliées de la culture québécoise: les archives religieuses. 47(2), 59-76.

DAVID, F. Les centres et les services d'archives du Québec se font tirer le portrait... statistique. Quelques considérations méthodologiques et résultats d'une préenquête. 47(1), 125-152.

ÉGLISE CATHOLIQUE DE QUÉBEC. Mémoire présenté dans le cadre de la consultation publique sur le renouvellement de la politique culturelle du Québec. 47(2), 77-90. 
GADOURY, N. Acquérir, évaluer et conserver des photographies dans un milieu de santé et de services sociaux. 47(1), 153-183.

GUITARD, L. A. [Compte rendu de l'ouvrage Les Ursulines de Québec. Espaces et mémoires de C. Cheyrou]. 47(1), 193-198.

LE CLECH, L. Archives et géographie: typologie, caractéristiques et perspectives. 47(1), 59-83.

MAUREL, D. Les centres et les services d'archives du Québec se font tirer le portrait... statistique. Quelques considérations méthodologiques et résultats d'une préenquête. 47(1), 125-152.

MICHON, P. Archivistique, histoire et Web sémantique: une approche interdisciplinaire basée sur l'événementiel. 47(1), 85-105.

OUELLET, D. Les débuts de la prise en charge de la gestion des documents administratifs au Centre intégré universitaire de santé et de services sociaux du Saguenay-Lac-Saint-Jean. 47(2), 21-42.

OURY, C. L'archivage du Web: bibliothèques et archives à la croisée des chemins. 47(1), 107-124.

PARK, E. G. La subjectivité dans la numérisation: les perspectives des professionnels. 47(1), 31-58.

PICHÉ, G. [Compte rendu de l'ouvrage Managing Active Business Records ( $2^{\mathrm{e}}$ édition) d'A. Bennick et J. Vasek Sitton]. 47(1), 199-202.

RAJOTTE, D. [Compte rendu de I'ouvrage Is Digital Different: How Information Creation, Capture, Preservation and Discovery are being Transformed de M. S. Moss, B. Endicott-Popovsky et M. J. Dupuis]. 47(1), 203-207.

REGROUPEMENT DES ARCHIVISTES RELIGIEUX DU QUÉBEC (RAR). Forces vives oubliées de la culture québécoise: les archives religieuses. 47(2), 59-76.

ROWAT, T. Les centres et les services d'archives du Québec se font tirer le portrait... statistique. Quelques considérations méthodologiques et résultats d'une préenquête. 47(1), 125-152. 
SIROIS, E. Introduction aux mémoires sur le renouvellement de la politique culturelle du Québec (2016). 47(2), 43-48.

TABLE DE CONCERTATION DES ARCHIVES RELIGIEUSES DE LA RÉGION DE MONTRÉAL (TCARM). Forces vives oubliées de la culture québécoise: les archives religieuses. 47(2), 59-76.

ZWARICH, N. Les centres et les services d'archives du Québec se font tirer le portrait... statistique. Quelques considérations méthodologiques et résultats d'une préenquête. 47(1), 125-152.

\section{INDEX DES SUJETS}

\section{Diffusion des archives}

CARTIER, F. Le traitement et la diffusion du fonds Armand-Frappier (ou comment être opportuniste en huit leçons). 47(2), 7-20.

\section{Documents sonores, audiovisuels et photographiques}

GADOURY, N. Acquérir, évaluer et conserver des photographies dans un milieu de santé et de services sociaux. 47(1), 153-183.

\section{Gestion d'un service d'archives et gestion des archives}

ZWARICH, N., MAUREL, D., LEMELIN, P., BAILLARGEON, D., DAVID, F. et ROWAT, $T$. Les centres et les services d'archives du Québec se font tirer le portrait... statistique. Quelques considérations méthodologiques et résultats d'une préenquête. 47(1), 125-152.

\section{Gouvernance et gestion des documents}

ALAOUI, S. La normalisation et la gestion intégrée des documents (GID): quelle relation? Réflexion sur les normes ISO 30300, ISO 30301, ISO 14641 et leur apport à l'implantation des systèmes de GID. 47(1), 7-30.

OUELLET, D. Les débuts de la prise en charge de la gestion des documents administratifs au Centre intégré universitaire de santé et de services sociaux du Saguenay-Lac-Saint-Jean. 47(2), 21-42. 


\section{Histoire, patrimoine et mémoire}

ASSOCIATION DES ARCHIVISTES DU QUÉBEC (AAQ). Renouvellement de la politique culturelle du Québec. 47(2), 49-58.

COMITÉ DES ARCHIVES DU CONSEIL DU PATRIMOINE RELIGIEUX DU QUÉBEC (CPRQ), REGROUPEMENT DES ARCHIVISTES RELIGIEUX DU QUÉBEC (RAR) et TABLE DE CONCERTATION DES ARCHIVES RELIGIEUSES DE LA RÉGION DE MONTRÉAL (TCARM). Forces vives oubliées de la culture québécoise: les archives religieuses. 47(2), 59-76.

ÉGLISE CATHOLIQUE DE QUÉBEC. Mémoire présenté dans le cadre de la consultation publique sur le renouvellement de la politique culturelle du Québec. 47(2), 77-90.

LE CLECH, L. Archives et géographie: typologie, caractéristiques et perspectives. 47(1), 59-83.

MICHON, P. Archivistique, histoire et Web sémantique: une approche interdisciplinaire basée sur l'événementiel. 47(1), 85-105.

SIROIS, E. Introduction aux mémoires sur le renouvellement de la politique culturelle du Québec (2016). 47(2), 43-48.

\section{Numérisation}

CHALIFOUR, J. et PARK, E. G. La subjectivité dans la numérisation: les perspectives des professionnels. 47(1), 31-58.

\section{Préservation numérique}

OURY, C. L'archivage du Web: bibliothèques et archives à la croisée des chemins. 47(1), 107-124.

\section{INDEX DES OUVRAGES RECENSÉS}

BENNICK, A. et VASEK SITTON, J. (2014). Managing Active Business Records (2e édition). Overland Park, KS: ARMA International, 248 p. (Compte rendu par G. Piché, 47(1), 199-202) 
CHEYROU, C. (2015). Les Ursulines de Québec. Espaces et mémoires. Québec, Québec: Fides, 211 p. (Compte rendu par L. A. Guitard, 47(1), 193-198)

MONNIER, S. et FIORENTINO, K. (dir.). (2016). Archives hospitalières. Regards croisés. Paris, France: L'Harmattan, 188 p. (Compte rendu par J. Bréard, 47(2), 95-98.)

MOSS, M. S., ENDICOTT-POPOVSKY, B. et DUPUIS, M. J. (dir.). (2015). Is Digital Different: How Information Creation, Capture, Preservation and Discovery are being Transformed. Londres, Angleterre: Facet Publishing, 217 p. (Compte rendu par D. Rajotte, 47(1), 203-207)

RASCAROLI, L., YOUNG, G. et MONAHAN, B. (2014). Amateur Filmmaking: The Home Movie, the Archive, the Web. New York, NY: Bloomsbury, 375 p. (Compte rendu par S. Brochu, 47(1), 185-191)

ROBERGE, M. (2016). La gestion intégrée des documents d'activité (GID) technologiques et en format papier. Québec, Québec: Éditions Michel Roberge, 400 p. (Compte rendu par S. Alaoui, 47(2), 91-94.) 